\title{
Non-invasive Myocardial Performance Mapping Using 3D Echocardiographic Stress-Strain Loops
}

\author{
João Pedrosa ${ }^{1}$, Jürgen Duchenne ${ }^{2}$, Sandro Queirós ${ }^{1,3,4}$, Ganna \\ Degtiarova $^{5}$, Olivier Gheysens ${ }^{5}$, Piet Claus ${ }^{1}$, Jens-Uwe Voigt ${ }^{2}$ \\ and Jan D'hooge ${ }^{1}$ \\ ${ }^{1}$ Laboratory on Cardiovascular Imaging and Dynamics, Department of \\ Cardiovascular Sciences, KU Leuven, Belgium \\ ${ }^{2}$ Clinical Cardiology, Department of Cardiovascular Sciences, KU Leuven, Belgium \\ ${ }^{3} \mathrm{ICVS} / 3 \mathrm{Bs}$ - PT Government Associate Laboratory, Braga/Guimarães, Portugal \\ ${ }^{4}$ Life and Health Sciences Research Institute (ICVS), School of Medicine, University \\ of Minho, Braga, Portugal \\ ${ }^{5}$ Department of Nuclear Medicine and Molecular Imaging, KU Leuven, Belgium \\ E-mail: jan.dhooge@kuleuven.be
}

April 2019

\begin{abstract}
Regional contribution to left ventricular ejection is of much clinical importance but its assessment is notably challenging. While deformation imaging is often used, this does not take into account loading conditions. Recently, a method for intraventricular pressure estimation was proposed, thus allowing for loading conditions to be taken into account in a non-invasive way. In this work, a method for 3D automatic myocardial performance mapping in echocardiography is proposed by performing 3D myocardial segmentation and tracking, thus giving access to local geometry and strain. This is then used to assess local left ventricular stress-strain relationships which can be seen as a measure of local myocardial work. The proposed method was validated against 18F-fluorodeoxyglucose positron emission tomography, the reference method to clinically assess local metabolism. Averaged over all patients, the mean correlation between FDG-PET and the proposed method was $0.67 \pm 0.18$. In conclusion, stressstrain loops were, for the first time, estimated from $3 \mathrm{D}$ echocardiography and correlated to the clinical gold standard for local metabolism, showing the future potential of RT3DE for the assessment of local metabolic activity of the heart.
\end{abstract}

Keywords: real-time $3 D$ echocardiography, performance mapping, myocardial work, Bspline explicit active surfaces 


\section{Introduction}

The assessment of the regional contribution to left ventricular (LV) ejection has long been an object of interest in clinical cardiology and is clinically most often assessed through visual wall motion readings. However, such readings are inherently subjective and prone to high intra- and inter-observer variability [1, 2]. More objective measurements of shortening indices through regional deformation imaging have thus been introduced to tackle this problem [3, 4, 5]. However, local deformation is highly dependent on loading conditions and to truly estimate myocardial performance, the relation between deformation and loading conditions must be taken into account [6]. Nevertheless, it is challenging to estimate loading conditions non-invasively and most studies thus resort to invasive intraventricular pressure measurements. Suga first proposed such an approach by successfully correlating LV pressure-volume loop area to global myocardial oxygen consumption in a canine model [7] and this approach was later validated in a clinical setting [8]. Following the same rationale, regional myocardial work was estimated by relating local myocardial deformation to pressure in several studies $[9,10,11,12,13]$. While these techniques have been proposed already decades ago, they have seen limited clinical application due to their complexity and the need for an invasive intraventricular pressure measurement. Recently, Russel et al. have proposed a method for non-invasive estimation of intraventricular pressure [14], thus allowing for non-invasive estimation of myocardial work. Nevertheless, pressure remains a global loading parameter as local loading, expressed as myocardial stress, depends on LV geometry. Such an approach was followed in a recent study [15], where the authors used Laplace's law to estimate local stress from non-invasive pressure measurements and local geometry, which, related to local strain was equated to local myocardial work.

In spite of these recent advances, the methodology remains complex as obtaining the full myocardial performance map requires 3 different long axis (LAx) views to be acquired and in each of the views the LV must be segmented and local strains computed. As such, the use of real-time 3D echocardiography (RT3DE) can play a role in decreasing the complexity of this method by decreasing the number of acquisitions needed. Although manual analysis of 3D images is more challenging than 2D, automatic methods can be used to obtain 3D local LV geometry and strains, further decreasing the complexity of the method. Moreover, the acquisition of RT3DE rather than 2D echocardiography (2DE) allows the analysis of the full 3D myocardial motion rather than being limited to in-plane motion as in previous studies $[12,14,15,16]$ where only longitudinal strain-stress loop area was considered.

As such, in this study, a method for non-invasive myocardial performance mapping on RT3DE is proposed. A fully automatic method for myocardial segmentation and tracking was used to obtain 3D local LV geometry and strain. Intraventricular pressure was estimated according to the method by Russel et al. [14], which is used together with local geometry to estimate local LV stress. The proposed LV stress-strain loop areas were then validated against $18 \mathrm{~F}$-fluorodeoxyglucose positron emission tomography 
(FDG-PET), the reference method to clinically assess local metabolism.

\section{Materials and Methods}

\subsection{D Left Ventricular Myocardial Segmentation}

3D LV myocardial segmentation was performed using B-spline Explicit Active Surfaces (BEAS) [17]. The key concept of BEAS is to regard the boundary of an object as an explicit function, where one of the coordinates of the points on the surface, $\mathbf{x}=\left\{x_{1}, \ldots, x_{n}\right\}$, is given explicitly as a function of the remaining coordinates, i.e. $x_{1}=\psi\left(x_{2}, \ldots, x_{n}\right)$. In this framework, $\psi$ is defined as a linear combination of B-spline basis functions:

$$
x_{1}=\psi\left(x_{2}, \ldots, x_{n}\right)=\psi\left(\mathbf{x}^{*}\right)=\sum_{\mathbf{k} \in \mathbb{Z}^{n-1}} c[\mathbf{k}] \beta^{d}\left(\frac{\mathbf{x}^{*}}{h}-\mathbf{k}\right),
$$

where $\mathbf{x}^{*}$ is the point of coordinates $\left\{x_{2}, \ldots, x_{n}\right\}$ and $\beta^{d}(\cdot)$ the uniform $(n-1)$-dimensional $\mathrm{B}$-spline of degree $d$. The knots of the B-splines are located on a rectangular grid defined on the chosen coordinate system, with a regular spacing given by $h$. The coefficients of the B-spline representation are gathered in $c[\mathbf{k}]$.

It was shown by Pedrosa et al. that the most efficient way to represent the myocardium using the BEAS framework was to perform a two-step (2S) approach where the endocardial segmentation was performed independently, followed by the epicardial segmentation represented as the thickness of the myocardium [18]. This allowed the epicardium to take advantage of the information from the endocardial segmentation without the need for extensive and complicated constraints between the two surfaces. This same representation was chosen for the current study. As in previous implementations for LV and myocardial segmentation, the angular discretization was set to $24 \times 16$ (elevation $\times$ azimuth) and the B-spline scale to $2^{1}$.

The evolution of the model is defined by the minimization of an energy criterion $E$. This energy is expressed by the sum of the data attachment term $E_{d}$ and a regularization term $E_{r}$ :

$$
E=E_{d}+E_{r} .
$$

The data attachment energy function $E_{d}$ follows a variation of the localized Yezzi energy adapted for endocardial segmentation proposed by Barbosa et al. [19].

To give information on the shape variations of the myocardium, a statistical shape model (SSM) was constructed using 289 cardiac magnetic resonance imaging datasets from the DOPPLER-CIP study [20]. The endo- and epicardium were contoured at ED and ES on both LAx and short axis (SAx) slices and an iterative closest point algorithm [21] was used to correct for misalignment between the slices. A 3D mesh was then interpolated from the aligned 2D contours at both time points. A more detailed description of this methodology can be found in the study by Pedrosa et al. [22].

As in the original study by Pedrosa et al. [22], the SSM was built in the BEAS coordinate system. The SSM shapes will then be represented through their B-spline 


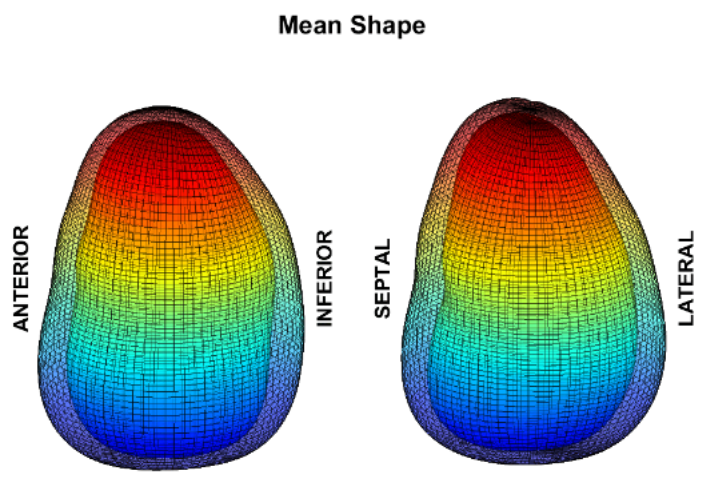

(a)

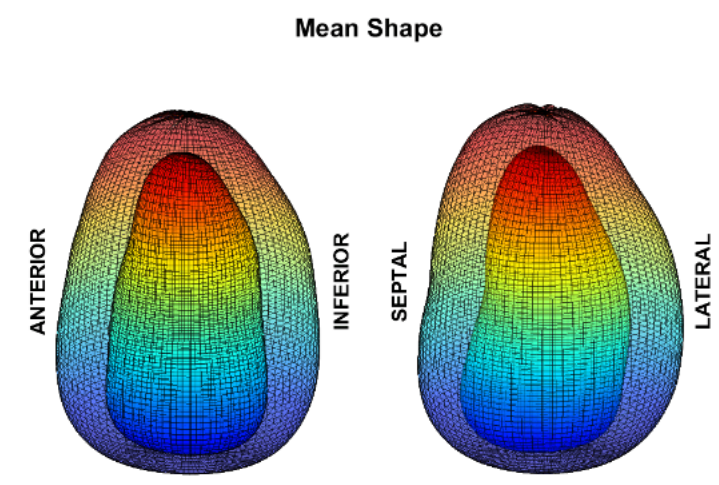

(b)

Figure 1: SSM mean models at ED (a) and ES (b).

representation coefficients $c[\mathbf{k}]$. Singular value decomposition [23] was used to obtain the eigenvectors $\mathbf{p}_{i}$ through which any shape from the dataset can be approximated according to:

$$
c[\mathbf{k}] \approx \bar{c}[\mathbf{k}]+\mathbf{P b}
$$

where $\mathbf{P}$ is the matrix of the first $t$ eigenvectors and $\mathbf{b}$ is a vector of $t$ weights which for any given shape corresponds to $\mathbf{b}=\mathbf{P}^{T}(c[\mathbf{k}]-\bar{c}[\mathbf{k}])$.

Given the 2S approach chosen for the myocardial segmentation, two different SSMs are needed at ED and ES, one representing the endocardium for the first segmentation step and a second representing both the endocardium and the myocardial thickness for the second segmentation step. In this way, the first segmentation step remains fully independent of the epicardium, while the second segmentation step derives information from the endocardial shape to obtain clues about the expected epicardial surface. As such, the endocardial SSM was built from the endocardial B-spline coefficients $c[\mathbf{k}]$, while the myocardial SSM was built by concatenating both the endocardial and the myocardial thickness B-spline coefficients so that the shape variations regarding both surfaces are modelled together. The mean shapes for each of these models are shown in Figure 1. Note that since these models are scaled only shape variations can be observed in this figure.

The SSM-based regularization of BEAS was performed according to two regularization energies, hard and soft, as proposed by Queirós et al. [24]. The hard term restricts the segmented shape to the shape variability observed in the training set, while the soft term penalizes high values of $b_{i}$, following the rationale that it is much more probable to find an average shape than a shape which is close to the variability limits. The regularization term $E_{r}$ is thus defined as

$$
E_{r}=\alpha E_{\text {hard }}+\beta E_{\text {soft }},
$$

where $\alpha$ and $\beta$ are hyperparameters controlling the relative weight between the two terms. 


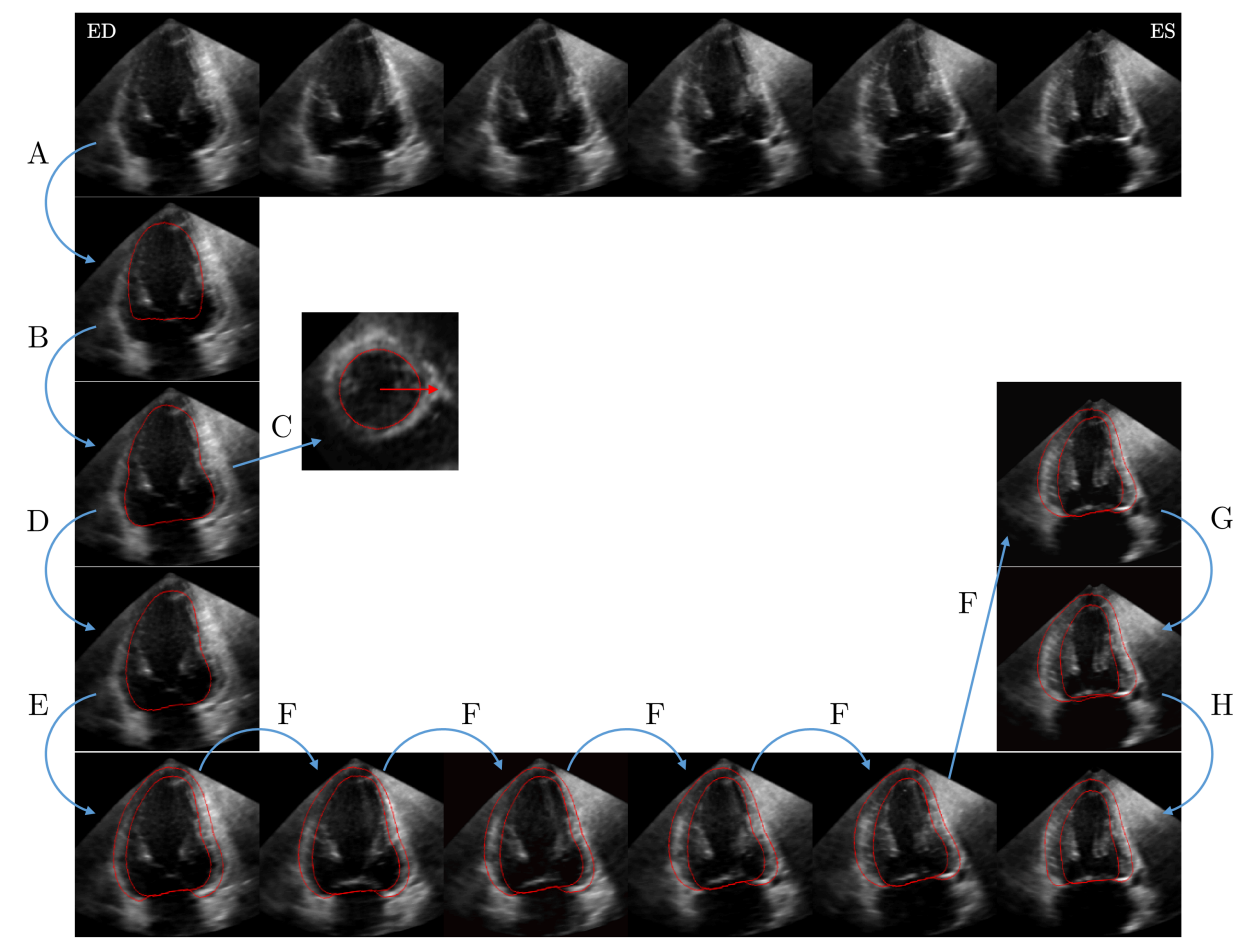

Figure 2: Conceptual description of the proposed segmentation and tracking framework.

A conceptual description of the LV myocardial segmentation framework is shown in Figure 2. First, automatic initialization is applied to the ED frame (Fig. 2-A). The first stage of endocardial segmentation is then performed using BEAS (Fig. 2B). The result from this segmentation is used to detect the SAx orientation (Fig. 2C) and this information is used to perform the second stage of segmentation using BEAS and the ED SSM (Fig. 2-D). The epicardial surface is then initialized and the ED myocardial SSM is used to perform the myocardial segmentation (Fig. 2-E). The final ED myocardial segmentation is then propagated frame to frame using a localized anatomical affine optical flow (lAAOF) strategy [25] (Fig. 2-F) and a final refinement of the ES segmentation is performed using first the ES endocardial SSM (Fig. 2-G) and then the ES myocardial SSM (Fig. 2-H).

To extract local geometry and strain, the LV segmentation obtained was divided into 17 segments according to Cerqueira et al. [26] and the extracted parameters averaged within each segment. At each frame, the local wall thickness $h$ was computed, defined as the distance from the endo- to the epicardial surface along the endocardial surface normal. Longitudinal and circumferential curvatures, $k_{l}$ and $k_{c}$, were also extracted taking advantage of the spherical domain on which the BEAS segmentation is defined:

$$
\begin{aligned}
& k_{l}=\bar{k}-\sqrt{\bar{k}^{2}-K}, \\
& k_{c}=\bar{k}+\sqrt{\bar{k}^{2}-K},
\end{aligned}
$$

where $\bar{k}$ and $K$ are respectively the mean and Gaussian curvatures obtained according 
to

$$
\begin{aligned}
& \bar{k}=\frac{e G+g E}{2 E G}, \\
& K=\frac{e g-f^{2}}{E G},
\end{aligned}
$$

where $E$ and $G$ are coefficients of the first fundamental form and $e, f$ and $g$ are coefficients of the second fundamental form [27]. Segmental strain was computed throughout the heart cycle for all three components - longitudinal $\left(\varepsilon_{l}\right)$, circumferential $\left(\varepsilon_{c}\right)$ and radial $\left(\varepsilon_{r}\right)$.

\subsection{Left Ventricular Stress Estimation}

In order to estimate LV stress, the equations proposed by Mirsky et al. [28] were used, thus:

$$
\begin{aligned}
\sigma_{l} & =\frac{P \cdot r_{l}}{h\left(2+\frac{h}{r_{l}}\right)}, \\
\sigma_{c} & =\frac{P}{h\left(\frac{1}{r_{c}}+\frac{1}{r_{l}}+\frac{h}{r_{l} r_{c}}\right)},
\end{aligned}
$$

where $\sigma_{l}$ and $\sigma_{c}$ are, respectively, the longitudinal and circumferential components of LV stress, $P$ is the intraventricular pressure and $r_{i}=1 / k_{i}$ is the radius of curvature along direction $i$.

Intraventricular pressure was estimated according to the method by Russel et al. [14], which essentially scales a typical LV pressure trace based on valve opening/closing and measured systolic arterial cuff pressure. The valve events were manually annotated by visual inspection of the RT3DE images.

To estimate the radial LV stress $\sigma_{r}$, the generalized 3D Hooke's law was used, assuming that the myocardium is an elastic, isotropic and incompressible medium:

$$
\varepsilon_{r}=\frac{1}{E}\left(\sigma_{r}-\mu\left(\sigma_{l}+\sigma_{c}\right)\right)
$$

where $E$ and $\mu$ are, respectively, the Young's modulus and Poisson coefficient of the medium. Given that the myocardium is assumed to be incompressible, $\mu=0.5$.

\subsection{Myocardial Performance Mapping}

To obtain a map of myocardial performance, a stress-strain loop was calculated for each of the components (i.e. radial, longitudinal and circumferential). The sum of the area of each of these loops, hereinafter referred to as $\mathrm{L}+\mathrm{C}+\mathrm{R}$ loop area, was then taken as an estimate of the total local myocardial work. 
It can be shown that the radial stress-strain loop area is independent of the Young's modulus $E$ chosen given that:

$$
\begin{aligned}
A\left\{\varepsilon_{r}, \sigma_{r}\right\} & =\frac{1}{2} \int \sigma_{r} \varepsilon_{r}{ }^{\prime}-\varepsilon_{r} \sigma_{r}{ }^{\prime} d t \\
& =\frac{1}{2} \int\left(E \varepsilon_{r}+\mu\left(\sigma_{l}+\sigma_{c}\right)\right) \varepsilon_{r}{ }^{\prime}-\varepsilon_{r}\left(E \varepsilon_{r}+\mu\left(\sigma_{l}+\sigma_{c}\right)\right)^{\prime} d t \\
& =\frac{\mu}{2} \int \sigma_{l} \varepsilon_{r}{ }^{\prime}-\varepsilon_{r} \sigma_{l}{ }^{\prime} d t+\frac{\mu}{2} \int \sigma_{c} \varepsilon_{r}{ }^{\prime}-\varepsilon_{r} \sigma_{c}{ }^{\prime} d t \\
& =\mu A\left\{\varepsilon_{r}, \sigma_{l}\right\}+\mu A\left\{\varepsilon_{r}, \sigma_{c}\right\},
\end{aligned}
$$

where $A\left\{\varepsilon_{i}, \sigma_{j}\right\}$ is the area of the loop formed by stress component $i$ and strain component $j$. For simplicity sake, the prime symbol was used to represent the derivative in $t$.

\section{Experiments}

Eleven non-ischemic and seven ischemic patients were selected from the WORKCRT study, which followed heart failure patients undergoing cardiac resynchronization therapy (CRT) at the University Hospitals Leuven (ClinicalTrials.gov Identifier: NCT02537782). The study was approved by the regional ethical committee (study number S5825), and all subjects gave written informed consent prior to inclusion. The patients were selected based on showing acceptable spatiotemporal resolution and image quality in RT3DE (Vivid E9 and E95, GE Vingmed Ultrasound, Horten, Norway) and FDG-PET analysis (Biograph 16 HireZ, Siemens Healthcare, Forchheim, Germany) one week prior to CRT.

3D LV myocardial segmentation was performed for each patient and the myocardial performance maps estimated based on the method described above. A comparison to FDG-PET was performed by using the normalized tracer uptake (\%). The LV segment with the highest tracer uptake was used as a reference (100\%), and segmental values reported as percentages of this value.

For comparison to the proposed myocardial performance maps, pressure-strain loop areas as proposed in the study of Russel et al. [14] were also computed. Furthermore, to disentangle the contribution of each variable, motion, geometry and asynchrony parameters were correlated against FDG-PET uptake. Segmental peak systolic longitudinal, radial and circumferential strain were extracted by averaging

for each segment. Average thickness $h$ and longitudinal and circumferential radii of curvature $k_{l}$ and $k_{c}$ at ED and ES were also extracted. Time to onset of shortening, defined as the time from ED to the beginning of shortening in each of the segmental strain curves, was also computed as a measure of asynchrony.

Finally, a sensitivity analysis of the proposed methodology was performed relative to the annotation of the valve events and the standard pressure curve used. Each of the valve events - aortic valve opening (AVO), aortic valve closure (AVC) and mitral valve opening (MVO) - was deliberately changed to one frame before or after the annotated 


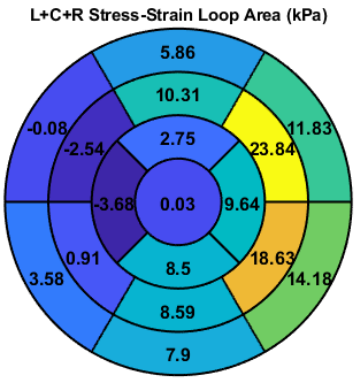

(a)

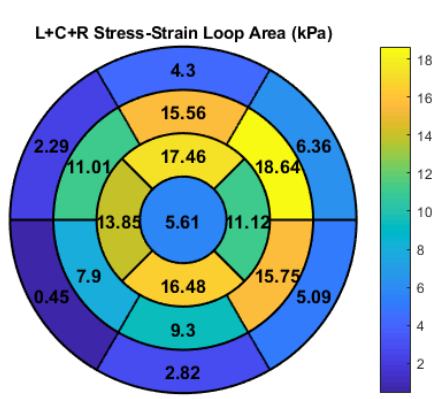

(d)

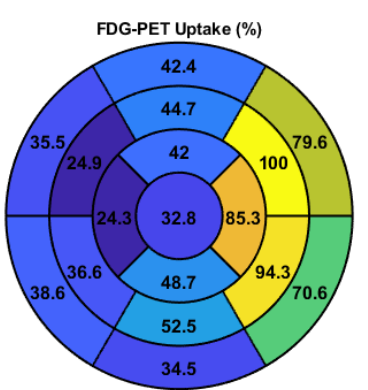

(b)

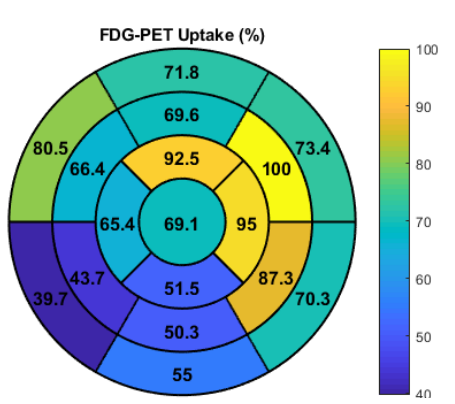

(e)

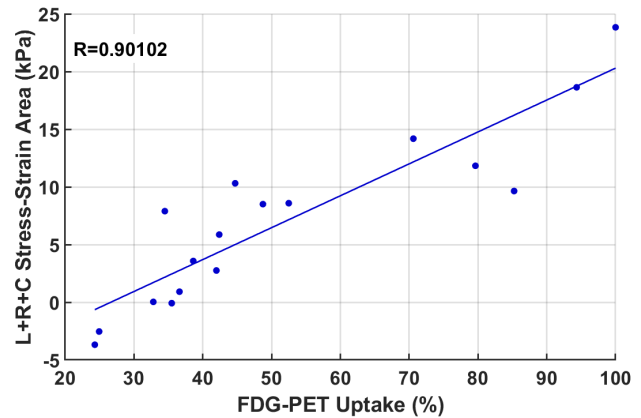

(c)

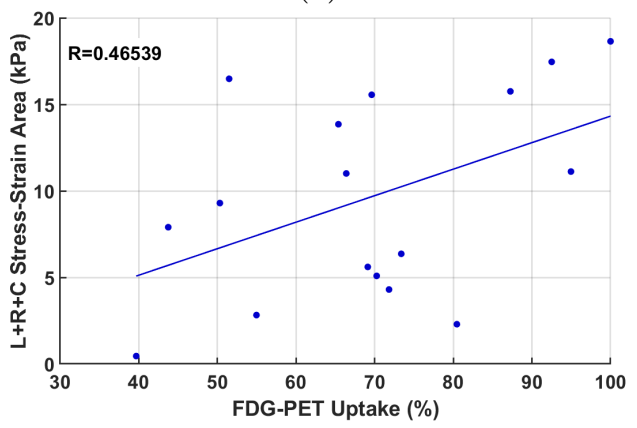

(f)

Figure 3: Myocardial performance mapping (a,d), FDG-PET tracer uptake (b,e) and correlation between the two (c,f) for patients with best (first row) and worst (second row) correlation to FDG-PET. In (a,b,d,e), mapping follows the 17-segment model [26] where the central segment represents the apical cap and each of the surrounding layers represent the apical, mid-cavity and basal segments. Regarding the circumferential location, starting from the topmost segment in a counterclockwise order are the anterior, anteroseptal, inferoseptal, inferior, inferolateral and anterolateral segments.

frames and the corresponding stress-strain loops were computed. Mitral valve closure was not tested because this event occurs on the first frame since the RT3DE images used were acquired using electrocardiography triggering. Secondly, the standard pressure curve proposed in [14] was linearly deformed to bring the peak systolic pressure forward by $100 \mathrm{~ms}$. Furthermore, the measured systolic pressure was deliberately changed by $5 \%$ to test the sensitivity to measurement error.

\section{Results}

Maps of myocardial performance and FDG-PET tracer uptake of two patients are shown in a 17-segment model [26] in Figure 3, as well as their respective correlations. Qualitatively, it can be observed that there is reasonable correspondence between the $\mathrm{L}+\mathrm{C}+\mathrm{R}$ stress-strain loop areas in Figure 3(a,d) and the FDG-PET maps in Figure $3(\mathrm{~b}, \mathrm{e})$. By plotting the segmental $\mathrm{L}+\mathrm{C}+\mathrm{R}$ stress-strain loop area values against the FDG-PET tracer uptake $(\%)$ as in Figure 3(c,f), a correlation value can be obtained showing the agreement between both measures. This value ranged from 0.47 (worst 


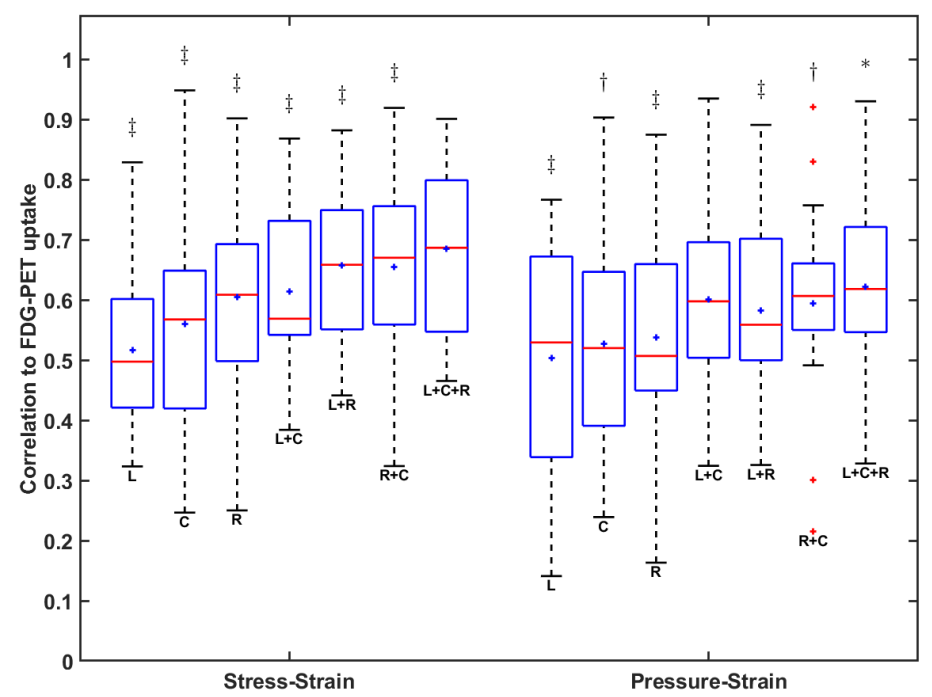

Figure 4: Correlation to FDG-PET tracer uptake across all patients for stress-strain and pressure-strain loop areas. L, C and $\mathrm{R}$ indicate the longitudinal, circumferential and radial components and the remaining boxplots are combinations of these. $\dagger$ and $\ddagger$ indicate significant differences at respectively $p<0.05$ and $p<0.01$ to the $\mathrm{L}+\mathrm{C}+\mathrm{R}$ data sample within each group. $*$ indicates significant differences at $p<0.05$ to $\mathrm{L}+\mathrm{C}+\mathrm{R}$ stress-strain loop area (tested only for $\mathrm{L}+\mathrm{C}+\mathrm{R}$ pressure-strain loop area). Box limits and the red line within each box represent the first, second (median) and third quartiles of the data and blue crosses represent the mean of the corresponding data sample. Whisker ends represent the last data point within 1.5 the interquartile range of the first or third quartile respectively and red crosses represent outliers outside this range.

case, Figure 3(f)) to 0.90 (best case, Figure 3(c)).

Fig. 4 shows the correlation to FDG-PET uptake of stress-strain and pressure-strain area loops for each spatial component and possible combinations $(\mathrm{L}+\mathrm{C}, \mathrm{L}+\mathrm{R}, \mathrm{R}+\mathrm{C}$ and $\mathrm{L}+\mathrm{C}+\mathrm{R})$. Because normalized FDG-PET uptake was used, direct comparison across patients is not possible and the results shown in Figure 4 are the average correlation across all patients according to the method used in the previous paragraph to obtain Figure $3(\mathrm{c}, \mathrm{f})$. It can be seen that for both stress-strain and pressure-strain loop areas the contribution of all three spatial components significantly outperforms any of the other individual components or combinations of two of these except for $\mathrm{L}+\mathrm{C}$ pressure-strain. Among all patients, $\mathrm{L}+\mathrm{C}+\mathrm{R}$ stress-strain has the best average correlation to FDG-PET uptake at $0.67 \pm 0.14$ and $\mathrm{L}+\mathrm{C}+\mathrm{R}$ pressure-strain has statistically significant inferior correlation at $p<0.05$. In non-ischemic patients $(N=11), \mathrm{L}+\mathrm{C}+\mathrm{R}$ stress-strain had an average correlation to FDG-PET uptake of $0.73 \pm 0.12$, whereas for ischemic patients $(N=7)$ the average correlation was $0.62 \pm 0.12$. The data distribution for each patient group is provided in the supplementary files.

Fig. 5 shows the correlation to FDG-PET uptake of peak systolic strain, myocardial thickness, longitudinal and circumferential radius of curvature and time to onset 


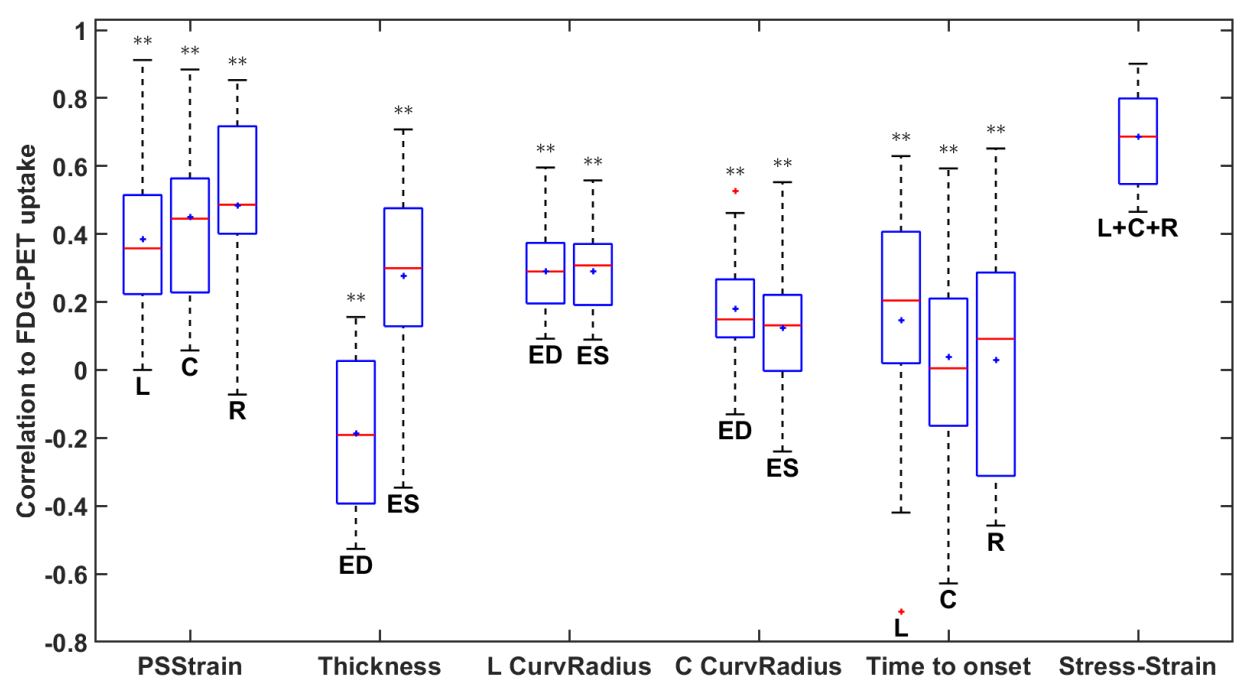

Figure 5: Correlation to FDG-PET tracer uptake across all patients for peak systolic strain (PSStrain), myocardial thickness, radii of curvature (CurvRadius) and time to onset. $\mathrm{L}+\mathrm{C}+\mathrm{R}$ stress strain loop area correlation is shown for comparison. L, C and R indicate the longitudinal, circumferential and radial components. $* *$ indicates significant differences at $p<0.01$ to $\mathrm{L}+\mathrm{C}+\mathrm{R}$ stress-strain loop area. Box plot format follows that of Figure 4.

of shortening. As in Figure 4, the boxplots represent distribution of per/patient correlations. It can be observed that among these measures, peak systolic strain correlates best to FDG-PET uptake. Nevertheless, all have statistically significant lower correlation when compared to $\mathrm{L}+\mathrm{C}+\mathrm{R}$ stress-strain area loop $(p<0.01)$.

Sensitivity to annotation of the valve events and pressure curve is shown in Figure 6. It can be seen that changing the timing of the annotated valve events (within one frame) has very little effect to the final result for any of the valve events. The temporal resolution of the RT3DE datasets, and thus the magnitude of error deliberately introduced to the timing of valve events, was $33 \pm 9 \mathrm{~ms}$. In regard to changes in the systolic pressure value it can be seen that these have no effect. The results obtained after replacement with a modified pressure curve with early peak systolic pressure are, however, significantly different $(p<0.05)$ than those obtained with the standard pressure curve but the overall magnitude of the correlation is unchanged.

In terms of computational time, the total time for ED segmentation was on average 3.6s whereas tracking took on average $0.8 \mathrm{~s} /$ frame (26s per dataset). Stressstrain loop area calculations took on average $17 \mathrm{~ms}$ totaling to about 30seconds to obtain the performance maps. All data was processed in a non-optimized MATLAB implementation.

\section{Discussion}

It is clear that a reasonable correlation to FDG-PET was obtained with the proposed 


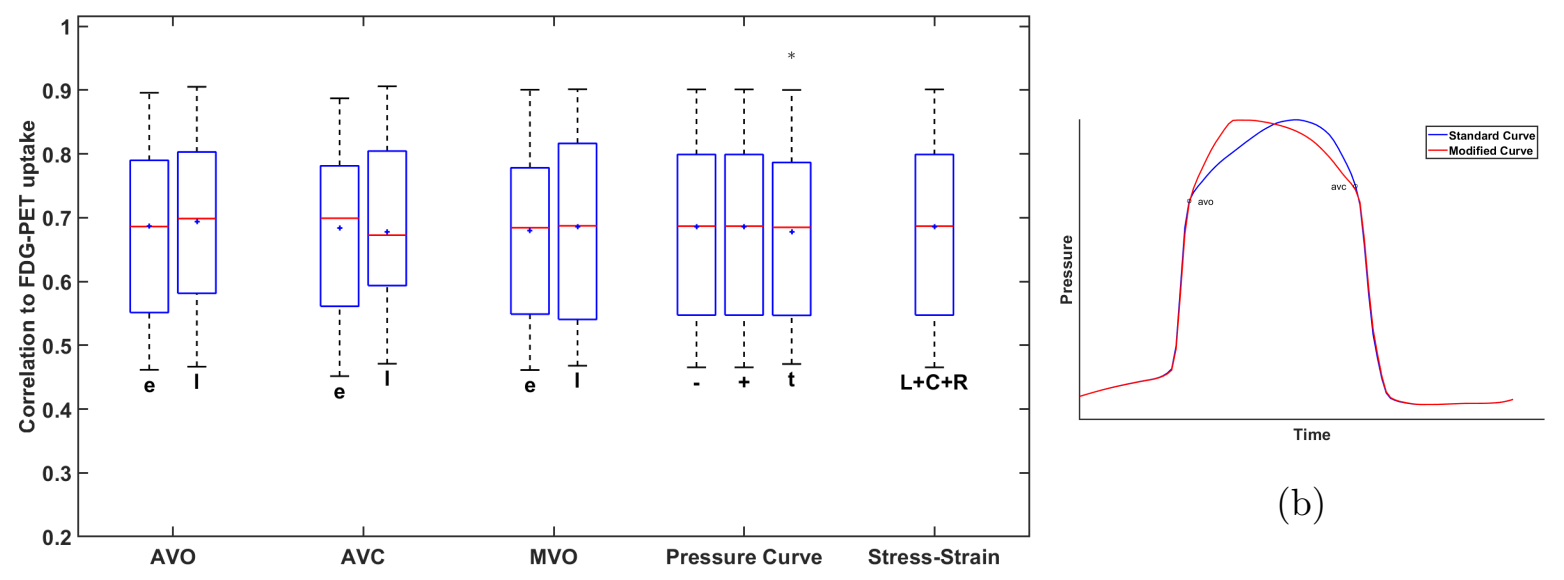

(a)

Figure 6: Sensitivity analysis to annotation of valve events and pressure curve. (a) Correlation to FDG-PET tracer uptake across all patients for deliberately changed valve event annotation and pressure curve. $\mathrm{L}+\mathrm{C}+\mathrm{R}$ stress strain loop area correlation is shown for comparison. Labels $e$ and $l$ stand for early and late annotation of the valve events by one frame respectively. Labels - and + stand for deviation of peak pressure value to $95 \%$ and $105 \%$ of original systolic pressure, whereas label $t$ stands for the replacement of the standard pressure curve by a modified curve. * indicates significant differences at $p<0.05$ to $\mathrm{L}+\mathrm{C}+\mathrm{R}$ stress-strain loop area. Box plot format follows that of Figure 4. (b) Standard pressure curve (blue) and modified curve with early peak systolic pressure.

methodology, which can then be regarded as a method for non-invasive LV myocardial work estimation. Both local deformation and loading conditions are taken into account, thus using additional information in comparison to myocardial deformation imaging, which is essential for the estimation of myocardial performance. The proposed method is also of low complexity, as only one image needs to be acquired. Moreover, the LV myocardial segmentation is fully automatic and only the valve events need to be annotated manually. In terms of computational complexity, the segmentation and tracking method represents by far the largest portion of time but the computational time for the segmentation could be significantly improved through optimization - it has been shown that $3 \mathrm{D}$ endocardial segmentation can be obtained using BEAS in $12.5 \mathrm{~ms}$ [17] in a $\mathrm{C}++$ implementation. It could thus be envisioned that this methodology can run on a scanner and be applied bedside.

Analysing Fig. 4, it becomes clear that the contribution of all three components of stress and strain is necessary in the final myocardial performance map, as the $\mathrm{L}+\mathrm{C}+\mathrm{R}$ loop area was statistically significantly better than any of the three components independently or combinations of two of the components. Specifically, the longitudinal stress-strain and pressure-strain loop areas are equivalent to the approaches of Cvijic et al. [15] and Russel et al. [14] as in those studies LAx 2DE was used and thus only longitudinal components were assessed. In spite of the promising results of those studies, it is shown that RT3DE can provide additional information, thereby resulting in better 
correlation to FDG-PET tracer uptake.

Furthermore, it was shown that the correlation between the $\mathrm{L}+\mathrm{C}+\mathrm{R}$ stress-strain and FDG-PET tracer uptake was statistically significantly different and greater than using $\mathrm{L}+\mathrm{C}+\mathrm{R}$ pressure-strain. This highlights the importance of considering local, rather than global loading, for an accurate myocardial performance map. Given that the only difference between the stress-strain and pressure-strain results shown here are the estimation of stress through the combination of global loading (pressure) and myocardial geometry for stress-strain loops, the importance of local loading considerations seems to be significant. The results of Figure 5 further highlight the contribution of loading, geometry, motion and asynchrony measures to the final result obtained with $\mathrm{L}+\mathrm{C}+\mathrm{R}$ stress-strain loop area. It is clear that $\mathrm{L}+\mathrm{C}+\mathrm{R}$ stress-strain, by combining local deformation and loading through geometry, can better correlate to FDG-PET than standard measures considered independently.

Looking specifically at the difference between ischemic and non-ischemic patients, it seems that the proposed methodology is more successful in non-ischemic patients. Nevertheless, no definite conclusions can be drawn given the reduced number of patients in this study and, if there would be a significant difference, it is not clear at this point what mechanism could lead to different results in these two groups of patients.

Nevertheless, there are limitations to this study which must be considered. First, the number of patients is relatively small and a larger dataset would certainly strenghten the conclusions drawn in this study. Second, given the fact that normalized FDG-PET tracer uptake was used as ground truth, a direct comparison between patients is not possible. This might have an effect on the final results as correlations are only possible within each patient. An alternative approach could be to use the FDG-PET standard uptake ratio as proposed by van den Hoff et al. [29], which would allow for a more robust measurement of myocardial oxygen consumption and a comparison across all patients. However, this method could not be applied to this population in the scope of this study.

Third, RT3DE is, in spite of the improvements in recent years, a more challenging technique than 2DE in terms of acquisition window and image quality in some patients, which might have an impact on the applicability of this method to the general population. Furthermore, variations in RT3DE image quality across patients will play a role on the accuracy of the results obtained. In spite of the robustness of the segmentation/tracking framework used in this study and demonstrated in $[18,22]$, it is expected that poor image quality will result in lower quality performance maps. Nevertheless, the improvements to RT3DE's spatiotemporal resolution are expected to continue as more advanced beamforming techniques migrate to commercial systems, improving the quality of information that can be obtained with RT3DE and simultaneously increasing the applicability of RT3DE based methods such as the one proposed in this study.

Fourth, the manual annotation of valve events might lead to interobserver variability. It was shown in Figure 6 that for the purpose of $\mathrm{L}+\mathrm{C}+\mathrm{R}$ stress-strain loop area calculations, small deviations in the selection of valve events (up to one 
RT3DE image frame) resulted only in nonsignificant changes to the resulting loop area. Nevertheless, the automation of the annotation of the valve events would be of interest as it is currently the only step that requires user interaction. This would remove all sources of interobserver variability and further simplify the method, rendering it fully automatic.

Finally, significant assumptions are necessary to estimate myocardial stress and this will influence the final results obtained. The methodology used to estimate intraventricular pressure is quite simplistic assuming a standard pressure curve, which will lead to deviations from the actual pressure curve in some patients. However, it has been shown in Figure 6 that reasonable modifications to the standard pressure curve lead to only limited changes in calculated area. Errors in the measurement of peak systolic pressure have, as expected, no effect on the correlation due to the fact that all segments are equally affected, having thus no effect on the correlation but only on the magnitude of myocardial work calculated. Changes to the pressure curve proved to have a significant effect on the correlation but the impact on the magnitude of the correlation was negligible. Furthermore, significant variations to the standard pressure curve are only expected in a small percentage of the population. Equations 9, 10 and 11 used to estimate LV stress also present numerous assumptions which are known to be violated in the LV myocardium. While numerous forms of these equations exist, the most well-known being perhaps Laplace's law, these were chosen as they predict different longitudinal and circumferential stress. Nevertheless, the assumptions involved, such as assuming an elastic, isotropic and incompressible myocardium, will inevitably bias the results obtained. As such, it would be important in the future to consider more complex analysis tools adapted to the complexity of the LV, such as finite element methods, where more adequate material laws can be incorporated. However, good correlation with FDG-PET, the reference method to clinically assess local metabolism, was obtained, validating the proposed method.

The correlation found between the proposed $\mathrm{L}+\mathrm{C}+\mathrm{R}$ stress-strain loop area and the current clinical gold standard to assess local metabolism (FDG-PET) highlights the importance of the proposed methodology. Given the use of ionising radiation and the higher cost of FDG-PET, the possibility of a simpler and safer technique, as is the case for ultrasound, would be extremely interesting in the clinical scenario. In spite of the limited validation provided by this study, the results obtained in this study are promising and motivate further research in this direction in the future. First, more extensive validation of this, or similar promising methods, is needed. This would provide further information on the applicability of this method to a larger population and would allow a more definite answer to the question of whether stress-strain loop area provides additional information when compared to the current methods. Secondly, the methodology itself could be improved. Automatic annotation of the valve events would be interesting to further automate the method. The integration of refinement tools for the automatic segmentation and tracking could also be interesting. Currently, 2DE deformation imaging is mostly done in a semi-automatic manner to ensure quality 
strain estimation. This technique could also be employed in RT3DE to ensure the quality of both strain and shape information retrieved, even in lower quality images. Nevertheless, the biggest challenge is the estimation of stress. The use of advanced methodologies such as finite element models is likely to give more accurate information on LV stress [30] and could have a significant impact on the correlation to FDG-PET uptake. The implementation of these techniques is, however, not straightforward in an automatic pipeline such as the proposed method, and would represent a significant burden in terms of computational complexity which is not the case for the current LV stress estimation.

\section{Conclusions}

In conclusion, a novel non-invasive method for myocardial performance mapping in RT3DE is proposed. The fact that the proposed method relies on RT3DE not only simplifies the protocol, as only one image must be acquired and processed, but gives access to the full 3D information, leading to more accurate results than previous similar implementations in 2DE. Furthermore, the proposed method relies on an automatic segmentation framework, where only the valve events must be manually annotated making it simple to apply.

Through the proposed technique, stress-strain loops were estimated from 3D echocardiography, which had not been previously reported in literature to the authors' knowledge. More importantly, the area of the stress-strain loops obtained correlated to the current clinical gold standard to assess local metabolism, FDG-PET, showing the future potential of RT3DE for local cardiac metabolism assessment.

\section{Acknowledgements}

This work was made possible through funding from the European Research Council under the European Union's Seventh Framework Program (HeartMAPAS; FP7/20072013/ERC Grant Agreement number 281748). 
Non-invasive Myocardial Performance Mapping

\section{Bibliography}

[1] Hoffmann R, Lethen H, Marwick T, Arnese M, Fioretti P, Pingitore A, Picano E, Buck T, Erbel R, Flachskampf F A et al. 1996 Journal of the American College of Cardiology 27 330-336

[2] Zhang Y, Chan A K, Yu C M, Yip G W, Fung J W, Lam W W, So N M, Wang M, Wu E B, Wong $\mathrm{J} \mathrm{T}$ et al. 2005 Journal of the American College of Cardiology 46 864-871

[3] D'hooge J, Heimdal A, Jamal F, Kukulski T, Bijnens B, Rademakers F, Hatle L, Suetens P and Sutherland G R 2000 European Journal of Echocardiography 1 154-170

[4] D'hooge J, Konofagou E, Jamal F, Heimdal A, Barrios L, Bijnens B, Thoen J, Van de Werf F, Sutherland G and Suetens P 2002 IEEE transactions on ultrasonics, ferroelectrics, and frequency control 49 281-286

[5] Elen A, Choi H F, Loeckx D, Gao H, Claus P, Suetens P, Maes F and D'hooge J 2008 IEEE transactions on medical imaging 27 1580-1591

[6] Rademakers F, Werf F, Mortelmans L, Marchal G and Bogaert J 2003 The Journal of physiology $546777-787$

[7] Suga H 1979 American Journal of Physiology-Heart and Circulatory Physiology 236 H498-H505

[8] Takaoka H, Takeuchi M, Odake M and Yokoyama M 1992 European heart journal 13 85-90

[9] Hisano R and Cooper G 1987 Circulation Research 61 318-328

[10] Tyberg J V, Forrester J S, Wyatt H L, Goldner S J, Parmley W W and Swan H J 1974 Circulation 49 748-754

[11] Forrester J S, Tyberg J V, Wyatt H L, Goldner S, Parmely W W and Swan H J 1974 Journal of applied physiology 37 771-775

[12] Urheim S, Rabben S I, Skulstad H, Lyseggen E, Ihlen H and Smiseth O A 2005 American Journal of Physiology-Heart and Circulatory Physiology 288 H2375-H2380

[13] Duchenne J, Claus P, Pagourelias E D, Mada R O, Puyvelde J V, Vunckx K, Verbeken E, Gheysens O, Rega F and Voigt J U 2018 Cardiology Journal ISSN 1897-5593

[14] Russell K, Eriksen M, Aaberge L, Wilhelmsen N, Skulstad H, Remme E W, Haugaa K H, Opdahl A, Fjeld J G, Gjesdal O et al. 2012 European heart journal 33 724-733

[15] Cvijic M, Duchenne J, Ünlü S, Michalski B, Aarones M, Winter S, Aakhus S, Fehske W, Stankovic I and Voigt J U 2017 European Heart Journal-Cardiovascular Imaging

[16] Duchenne J, Turco A, Ünlü S, Pagourelias E D, Vunckx K, Degtiarova G, Bézy S, Cvijic M, Nuyts J, Claus P et al. 2019 Circulation: Arrhythmia and Electrophysiology $\mathbf{1 2}$

[17] Barbosa D, Dietenbeck T, Schaerer J, D'hooge J, Friboulet D and Bernard O 2012 IEEE Transactions on Image Processing 21 241-251

[18] Pedrosa J, Barbosa D, Heyde B, Schnell F, Rösner A, Claus P and D'hooge J 2017 IEEE transactions on ultrasonics, ferroelectrics, and frequency control 64 525-536

[19] Barbosa D, Dietenbeck T, Heyde B, Houle H, Friboulet D, D'hooge J and Bernard O 2013 Ultrasound in Medicine and Biology 39 89-101

[20] Rademakers F, Engvall J, Edvardsen T, Monaghan M, Sicari R, Nagel E, Zamorano J, Ukkonen H, Ebbers T, Di Bello V, Voigt J U, Herbots L, Claus P and D'hooge J 2013 Scandinavian Cardiovascular Journal 47 329-334 ISSN 1651-2006

[21] Besl P J and McKay N D 1992 Method for registration of 3-D shapes Sensor Fusion IV: Control Paradigms and Data Structures vol 1611 (Int Society for Optics and Photonics) pp 586-607

[22] Pedrosa J, Queirós S, Bernard O, Engvall J, Edvardsen T, Nagel E and Dhooge J 2017 IEEE transactions on medical imaging 36 2287-2296

[23] Jolliffe I 2014 Principal Component Analysis (John Wiley \& Sons, Ltd) ISBN 9781118445112

[24] Queirós S, Papachristidis A, Barbosa D, Theodoropoulos K C, Fonseca J C, Monaghan M J, Vilaça $\mathrm{J} \mathrm{L}$ and D'hooge J 2016 IEEE transactions on medical imaging 35 2015-2025

[25] Queirós S, Vilaça J L, Morais P, Fonseca J C, D'hooge J and Barbosa D 2017 International journal for numerical methods in biomedical engineering 33 e2871

[26] Cerqueira M D, Weissman N J, Dilsizian V, Jacobs A K, Kaul S, Laskey W K, Pennell 
D J, Rumberger J A, Ryan T and Verani M S 2002 Journal of the American Society of Echocardiography $\mathbf{1 5} 463-467$

[27] Abbena E, Salamon S and Gray A 2006 Modern Differential Geometry of Curves and Surfaces with Mathematica, Third Edition Textbooks in Mathematics (Taylor \& Francis) ISBN 9781584884484

[28] Mirsky I, Corin W J, Murakami T, Grimm J, Hess O M and Krayenbuehl H P 1988 Circulation 78 68-80

[29] van den Hoff J, Oehme L, Schramm G, Maus J, Lougovski A, Petr J, Beuthien-Baumann B and Hofheinz F 2013 EJNMMI research 377

[30] Gsell M A, Augustin C M, Prassl A J, Karabelas E, Fernandes J F, Kelm M, Goubergrits L, Kuehne $\mathrm{T}$ and Plank G 2018 International journal for numerical methods in biomedical engineering $\mathbf{3 4}$ e3147 